\title{
Escala de Motivação Académica: Validação no Ensino Superior Público Português
}

\author{
Academic Motivation Scale: Validity in Portuguese Public Higher Education
}

Marco Ferreira Ribeiro ${ }^{1}$

(1D https://orcid.org/0000-0001-8405-3916

Vasco Saraiva ${ }^{1}$

(ib) https://orcid.org/0000-0002-8946-9651

Paulo Pereira ${ }^{1}$

(1) https://orcid.org/0000-0002-3941-8274

Célia Ribeiro ${ }^{1}$

(1D) https://orcid.org/0000-0002-1000-6890

Universidade Católica Portuguesa, Viseu, Portugal ${ }^{1}$ 


\title{
Resumo
}

Esta investigação teve como objetivo o estudo das propriedades psicométricas e posterior validação da Escala de Motivação Académica proposta por Guimarães e Bzuneck (2008), em Portugal, através da utilização de duas amostras, a Amostra A constituída por 568 estudantes do ensino superior público e a Amostra B constituída por 589 estudantes igualmente do ensino superior publico, com uma abrangência nacional. Para tratamento dos dados foram utilizados os softwares SPSS 25 e AMOS 25. Foram conduzidas duas análises fatoriais confirmatórias em cada amostra e uma análise de grupos múltiplos, sendo que os resultados apontaram para a validade da escala reespecificada na avaliação da motivação no ensino superior e sua robustez, com destaque para correlações elevadas entre as dimensões Motivação Extrínseca por Regulação Integrada e Motivação Intrínseca, Motivação Extrínseca, por Regulação Integrada e Motivação Extrínseca por Regulação Introjetada e por Motivação Extrínseca por Regulação Externa e Desmotivação. Por outro lado, verificou-se que o modelo de escala proposto é invariante.

Palavras-chave: motivação académica; escala; validação.

\begin{abstract}
This research aimed to study the psychometric properties and subsequent validation of the Academic Motivation Scale proposed by Guimarães and Bzuneck (2008), in Portugal using two samples, the sample A constituted by 568 students of public higher education and the sample B constituted by 589 public higher education students, with a national scope. SPSS 25 and AMOS 25 software were used for data processing. Two confirmatory factor analyzes were conducted in each sample and a multiple group analysis, and the results pointed to the validity of the re-specified scale of the motivation evaluation in higher education, especially high correlations between the dimensions Extrinsic Motivation by Integrated Regime and Intrinsic Motivation, Extrinsic Motivation by Integrated Regulation and Extrinsic Motivation by Introjected Regulation and by Extrinsic Motivation by External Regulation and Demotivation. It was verified that the proposed scale model is invariant.
\end{abstract}

Keywords: academic motivation; scale; validation.

JEL Code: A23, I2, D73. 


\section{Introdução}

O comportamento humano tem vindo a suscitar diversas investigações, nomeadamente ao nível da motivação. A motivação, termo proveniente do latim movere, pode ser definida como uma tensão afetiva suscetível de desencadear uma determinada atividade com o objetivo do alcance de um determinado objetivo/desejo (Eccheli, 2008), tendo vindo a ganhar cada vez mais relevância tanto no contexto científico e académico como a nível organizacional. Em especifico, a motivação ao nível do ensino superior tem sido destaque de diversas investigações (Davoglio, Santos, \& Lettnin, 2016; Leal, Miranda, \& Carmo, 2013; Núñez \& León, 2018; Ramos, 2013; Reboredo \& Monteiro, 2015), tendo como objetivo o estudo dos fatores que influenciam a motivação do estudante.

Neste sentido, a motivação no ensino superior surge como uma temática que tem vindo a ganhar cada vez mais relevância, dado o seu contributo para o desempenho académico (Cunha \& Carrilho, 2005). Um aluno orientado para a aprendizagem e para a busca por novos conhecimentos e desafios, isto é, um aluno motivado, terá um desempenho superior quando comparado com um aluno desmotivado (Cardoso \& Bzuneck, 2004). Por outro lado, associado ao estudo da motivação têm vindo a ser desenvolvidas várias escalas de avaliação da motivação no ensino superior dada a sua importância no contexto educacional, com destaque para Boruchovitch (2008); Davoglio, Santos e Lettnin (2016); Leal, Miranda e Carmo (2013); Martinelli e Bartholomeu (2007); Núñez e León (2018); Ramos (2013); Reboredo e Monteiro (2015); Rodrigues e Joly (2011) e Sobral (2003).

Face ao exposto, a presente investigação tem como objetivo o estudo das propriedades psicométricas da Escala de Motivação Académica, baseada na Teoria da Autodeterminação e proposta por Guimarães e Bzuneck (2008), em Portugal, bem como a sua validação em duas amostras do ensino superior português. Para além disso, este estudo apresenta ainda como objetivo o estudo das correlações existentes entre as dimensões presentes no instrumento e testa a invariância entre as amostras do modelo de escala proposto.Com base neste estudo verificou-se a validade da Escala de Motivação Académica e robustez através da condução de duas análises fatoriais confirmatórias em cada amostra e uma análise de grupos múltiplos. Verificou-se, neste sentido, a relevância de estudos de validação de escalas com vista ao seu aprimoramento teórico e a robustez da escala supracitada na avaliação da motivação académica.

\section{Teoria da Autodeterminação}

A Teoria da Autodeterminação foi desenvolvida em 1981 por Ryan e Deci com vista à perceção da qualidade e quantidade/intensidade da motivação, entendida como a energia, direção e persistência, como forma de alcançar o bem-estar psicológico a partir da autodeterminação (Deci \& Ryan, 1985; Menard, Bott, \& Crossler, 2017). No entanto, já em 1968 Porter e Lawer teriam proposto um modelo de motivação para o trabalho constituído por duas vertentes (Leal et al., 2013).

A Teoria da Autodeterminação inicialmente defendia a existência de duas necessidades básicas como forma de se sentir motivado: necessidade de competência (autoconfiança para a realização - Saber) e necessidade de autonomia (responsabilidade - Realizar) (Deci \& Ryan, 1985). Porém, o modelo evolui com o acréscimo de mais uma necessidade, a necessidade de relacionamento (conexão - Experimentar Estímulos), sendo estas três necessidades básicas essenciais ao bem-estar, no sentido de inibição de sentimentos de angústia e ao aumento da motivação intrínseca (Deci \& Gagné, 2005). Este tipo de motivação revela a gratificação e o interesse demonstrados pelo indivíduo ao exercer uma atividade por vontade própria e sua opção (Deci \& Gagné, 2005; Leal et al., 2013). Para além da motivação intrínseca, o modelo integrou mais dois tipos de motivação: desmotivação e motivação extrínseca (Deci \& Gagné, 2005; Leal et al., 2013; Menard et al., 2017; Rigby \& Ryan, 2018). 
A desmotivação refere-se à falta de intenção para agir, ocorrendo pela falta de competência para a realização da ação ou pela perceção de que a realização da mesma não permite obter os resultados desejados (Menard et al., 2017; Stover, Bruno, Uriel, \& Liporace, 2017). Por outro lado, na motivação extrínseca o indivíduo é levado à ação por condições que lhe são externas, o oposto da motivação intrínseca, sem sentir satisfação na ação que exerce (Deci \& Gagné, 2005), levando ao envolvimento no trabalho por razões instrumentais, de aprovação social, autoestima ou por um objetivo pessoal que valorize (Deci \& Gagné, 2005; Wolsink, Belschak, \& Hartog, 2018). Neste sentido, a motivação extrínseca é geralmente projetada por quatro tipos de regulação: externa, introjetada, identificada e integrada, dependendo do grau de autonomia ou controlo (Deci \& Gagné, 2005). No caso da regulação externa, esta é considerada a forma menos autónoma da motivação extrínseca estando a ação inerente a obter um resultado desejado (prestigio) ou medo de punição (Deci \& Gagné, 2005; Olafsen, Deci, \& Halvari, 2005; Sheehan, Herring, \& Campbell, 2018). Quanto à regulação introjetada, esta implica a realização de uma atividade que não está de acordo com os valores individuais do indivíduo (Deci \& Gagné, 2005; Gustafsson, Carlin, Podlog, Stenling, \& Lindwall, 2018; Vanhalst, Luyckx, Petegem, \& Soenens, 2018), ocorrendo quando o indivíduo adota padrões de autoestima e de aprovação social que não se identifica totalmente (Deci \& Gagné, 2005; Gustafsson et al., 2018). Nestes casos, o indivíduo tende a manifestar sentimentos positivos, como forma de evitar sentimentos negativos de indignidade, vergonha ou culpa (Gustafsson et al., 2018; Vanhalst et al., 2018). Quanto à regulação identificada, o indivíduo pode sentir-se autónomo mesmo quando externamente motivado, uma vez que este se identifica com o propósito da atividade (Castonguay \& Miquelon, 2018; Haerens et al., 2018; Sheehan et al., 2018). Neste sentido, tal implica a prévia avaliação das condições contextuais de forma a avaliar a conveniência da execução da ação (Deci \& Gagné, 2005; Rigby \& Ryan, 2018). Por último, a motivação extrínseca inclui ainda a regulação integrada que pressupõe que embora o indivíduo se identifique com o valor da atividade, esta não possui significado para si, pelo que este age de acordo com o que alcançará com essa ação (Núñez \& León, 2018; Sebire et al., 2018).

\section{Escala de Motivação Académica}

A Escala de Motivação Académica (EMA) resultou da tradução para português do Brasil por Sobral (2003) da Echelle de Motivation en Educacion (EME) elaborada e validada por Vallerand, Blais, Briere e Pelletier (1989) no Canadá. Estas escalas, a original e a traduzida, foram, assim, utilizadas por outras investigações, nomeadamente Boruchovitch (2008); Davoglio et al. (2016); Leal et al. (2013); Martinelli e Bartholomeu (2007); Núñez e León (2018); Ramos (2013); Reboredo e Monteiro (2015); Rodrigues e Joly (2011); Sobral (2003) e Vallerand et al. (1989), o que permitiu verificar níveis satisfatórios de consistência interna. Neste sentido, a utilização desta escala tem vindo a revelar-se crescente no contexto de investigação da motivação no ensino superior.

A escala original elaborada por Vallerand et al. (1989), como referido, assim como a traduzida por Sobral (2003) são constituída por um conjunto de vinte e oito itens avaliados por uma escala do tipo Likert, sendo esses itens subdivididos em sete subescalas compostas por quatro itens cada, de forma a avaliar três tipos de motivação intrínseca (Competência, Autonomia e Conexão) e três tipos de motivação extrínseca (Externa, Introjeção e Identificação) e Desmotivação (Vallerand, Blais, Briere, \& Pelletier, 1989; Sobra, 2003). A validade de um instrumento de medida deve ser continuamente validado através da utilização de diferentes amostras e em momentos diferentes, sendo que o aperfeiçoamento do instrumento de avaliação pode levar a um aprimoramento teórico (Guimarães \& Bzuneck, 2008). Neste sentido, pelo fato das escalas, a original e a traduzida, não contemplarem todas as dimensões propostas pela teoria anteriormente enunciada, nomeadamente, a dimensão motivação extrínseca por regulação integrada, e por Sobral (2003) concluir uma menor estabilidade temporal do instrumento e uma menor consistência interna face ao original, embora aceitável, Guimarães e Bzuneck (2008) desenvolveram uma adaptação da escala com vista ao seu aperfeiçoamento e aprimoramento teórico. Nesta adaptação levada a cabo por Guimarães e Bzuneck (2008), oito juízes integrantes do grupo de investigação sobre Motivação no Contexto Escolar - CNPq/UEL receberam a definição teórica para cada tipo de motivação e um conjunto de itens para que, entre estes, escolhessem os itens que mais se aproximassem do conceito. 
De acordo com Guimarães e Bzuneck (2008) os itens apresentados foram elaborados a partir da Teoria da Autodeterminação ou retirados do instrumento original traduzido por Sobral (2003). Neste sentido, de acordo com os investigadores supracitados, foram excluídos oito itens que descreviam a frequência do ensino superior como uma meta apontada para o futuro, foram formulados novos itens para avaliação da motivação extrínseca por regulação externa e identificada, foram formulados itens para avaliação da motivação extrínseca por regulação integrada e na avaliação da motivação intrínseca permaneceram apenas itens que abordavam emoções positivas relativas ao estudo no ensino superior.

\section{Metodologia}

A investigação empírica realizada apresenta como objetivo a avaliação das propriedades psicométricas da Escala de Motivação Académica (EMA) proposta por Guimarães e Bzuneck (2008), adaptada a português de Portugal, bem como a sua validação. O instrumento adaptado a português de Portugal é apresentado em anexo. Neste contexto, foi desenvolvida uma investigação de cariz quantitativo, descritivo e correlacional, através da recolha de dados com a utilização de um inquérito por questionário online aplicado durante os meses de maio e junho de 2018, tendo sido inicialmente testado num grupo constituído por 20 estudantes do ensino superior. Neste sentido, foram inquiridos 1.157 estudantes do ensino superior público português e constituídas duas amostras: Amostra A e Amostra B.

De acordo com Guimarães e Bzuneck (2008), o instrumento de avaliação referido anteriormente contempla 29 itens teoricamente agrupados em três itens da motivação intrínseca, quatro itens de Motivação Extrínseca por Regulação Integrada, quatro itens de Motivação Extrínseca por Regulação Identificada, seis itens de Motivação Extrínseca por Regulação Introjetada, seis itens de Motivação Extrínseca por Regulação Externa e seis itens de Desmotivação, tal como se pode verificar na Tabela 2. Como referido anteriormente, a agrupação dos itens por dimensão foi levado a cabo por Guimarães e Bzuneck (2008).

De acordo com Guimarães e Bzuneck (2008), cada item foi enquadrado numa proposição afirmativa e avaliado numa escala tipo Likert de sete categorias (de 1 - Nenhuma Correspondência a 7 - Total Correspondência). Para o tratamento dos dados obtidos, foram utilizados os programas estatísticos SPSS 25.0 e AMOS 25.0, tendo sido utilizado, ao longo da análise estatística um valor de referência de 5\%. Inicialmente foi descrita cada uma das amostras e apresentadas as respetivas estatísticas descritivas. Foi igualmente conduzida uma Análise Fatorial Confirmatória na Amostra A como forma de ajustamento do modelo proposto e validado na Amostra B. Por último, foi conduzida uma Análise de Grupos Múltiplos com o objetivo de testar a invariância do modelo proposto entre ambas as amostras (Kline, 2005; Marôco, 2010).

\section{Descrição das amostras}

Como anteriormente referido, nesta investigação foram constituídas de forma não probabilística duas amostras representativas da população em estudo (Amostra A e Amostra B) através da utilização de um processo conhecido como bola de neve, isto é, foi apresentado um pedido de colaboração para a divulgação do questionário por estudantes de licenciatura e mestrado integrado a diversas universidades e institutos politécnicos. Os inqueridos foram integrados na amostra A ou B de forma aleatória.

A amostra A envolveu 568 estudantes do ensino superior público português, 212 estudantes (37.3\%) do sexo masculino e 356 estudantes $(62.7 \%)$ do sexo feminino, de várias universidades a nível nacional de cursos de licenciatura, mestrado e mestrado integrado de várias áreas do conhecimento, desde as áreas de engenharia, ciências sociais, artes, linguística, ciências naturais, direito e filosofia. Neste sentido, foram inquiridos 84 indivíduos (14.8\%) da Universidade de Aveiro, 84 (14.8\%) da Universidade do Porto, 21 (3.7\%) da Universidade do Minho, 52 (9.2\%) da Universidade de Coimbra, $21(3.7 \%)$ da Universidade da Beira Interior, $59(10,4 \%)$ da Universidade de Trás-os-Montes e Alto 
Douro, 27 (4.8\%) da Universidade do Algarve, 35 (6.2\%) da Universidade de Évora, 9 (1.6\%) do Instituto Politécnico de Beja, 17 (3.0\%) da Universidade da Madeira, 51 (9.0\%) da Universidade dos Açores, 30 (5.3\%) do Instituto Politécnico de Castelo Branco, 64 (11.3\%) da Universidade de Lisboa e $14(2.5 \%)$ do Instituto Politécnico de Viseu. No geral, 169 estudantes (29.8\%) está inscrito no $1^{\circ}$ ano, $158(27.8 \%)$ no $2^{\circ}$ ano, $159(28.0 \%)$ no $3^{\circ}$ ano, $50(8.8 \%)$ no $4^{\circ}$ ano e $32(5.6 \%)$ no $5^{\circ}$ ano. Para além disso, 63 dos estudantes inquiridos (11.1\%) são trabalhadores estudantes. Verifica-se também uma idade média de 21,57 anos na amostra compreendida entre uma idade mínima de 17 anos e uma idade máxima de 59 anos. Quanto ao estado civil, 544 indivíduos (95.8\%) são solteiros, 12 (2.1\%) casados ou em união de factos e 4 (0.7) divorciados ou separados. Por último, 14 dos indivíduos inquiridos (2.5\%) tem filhos, o que não se verifica nos restantes 554 indivíduos (97.5\%) que compõem a amostra.

A amostra B envolveu 589 estudantes do ensino superior público português, 212 estudantes (36\%) do sexo masculino e 377 estudantes (64\%) do sexo feminino, de várias universidades a nível nacional de cursos de licenciatura, mestrado e mestrado integrado de várias áreas do conhecimento, desde as áreas de engenharia, ciências sociais, artes, linguística, ciências naturais, direito e filosofia. Neste sentido, foram inquiridos 85 indivíduos (14.4\%) da Universidade de Aveiro, 105 (17.8\%) da Universidade do Porto, 18 (3.1\%) da Universidade do Minho, 77 (13.1\%) da Universidade de Coimbra, 23 (3.9\%) da Universidade da Beira Interior, $58(9,8 \%)$ da Universidade de Trás-os-Montes e Alto Douro, 39 (6.6\%) da Universidade do Algarve, 45 (7.6\%) da Universidade de Évora, 10 (1.7\%) do Instituto Politécnico de Beja, 11 (1.9\%) da Universidade da Madeira, 26 (4.4\%) da Universidade dos Açores, 23 (3.9\%) do Instituto Politécnico de Castelo Branco, 53 (9\%) da Universidade de Lisboa e 16 (2.7\%) do Instituto Politécnico de Viseu. No geral, 171 estudantes (29\%) está inscrito no $1^{\circ}$ ano, 182 (30.9\%) no $2^{\circ}$ ano, $163(27.7 \%)$ no $3^{\circ}$ ano, $43(7.3 \%)$ no $4^{\circ}$ ano e $30(5.1 \%)$ no $5^{\circ}$ ano. Para além disso, 79 dos estudantes inquiridos (13.4\%) são trabalhadores estudantes. Verifica-se também uma idade média de 21.81 anos na amostra compreendida entre uma idade mínima de 17 anos e uma idade máxima de 47 anos. Quanto ao estado civil, 562 indivíduos (95.4\%) são solteiros, 20 (3.4\%) casados ou em união de factos e 3 (0.5) divorciados ou separados. Por último, 17 dos indivíduos inquiridos (2.9\%) tem filhos, o que não se verifica nos restantes 572 indivíduos (97.1\%) que compõem a amostra.

Tendo em conta a descrição das amostras, constatou-se não existir diferenças significativas entre as amostras apresentadas, atendendo aos resultados do teste do Qui-Quadrado.

\section{Resultados}

Atendendo aos itens que constituem a Escala de Motivação Académica (EMA), são apresntadas as estatísticas descritivas por cada item na Tabela 1. De notar que, os itens que correspondem a uma baixa motivação estão enquadrados na dimensão desmotivação apresentada na Tabela 2. 
Tabela 1

Estatísticas: Itens da Escala de Motivação Académica

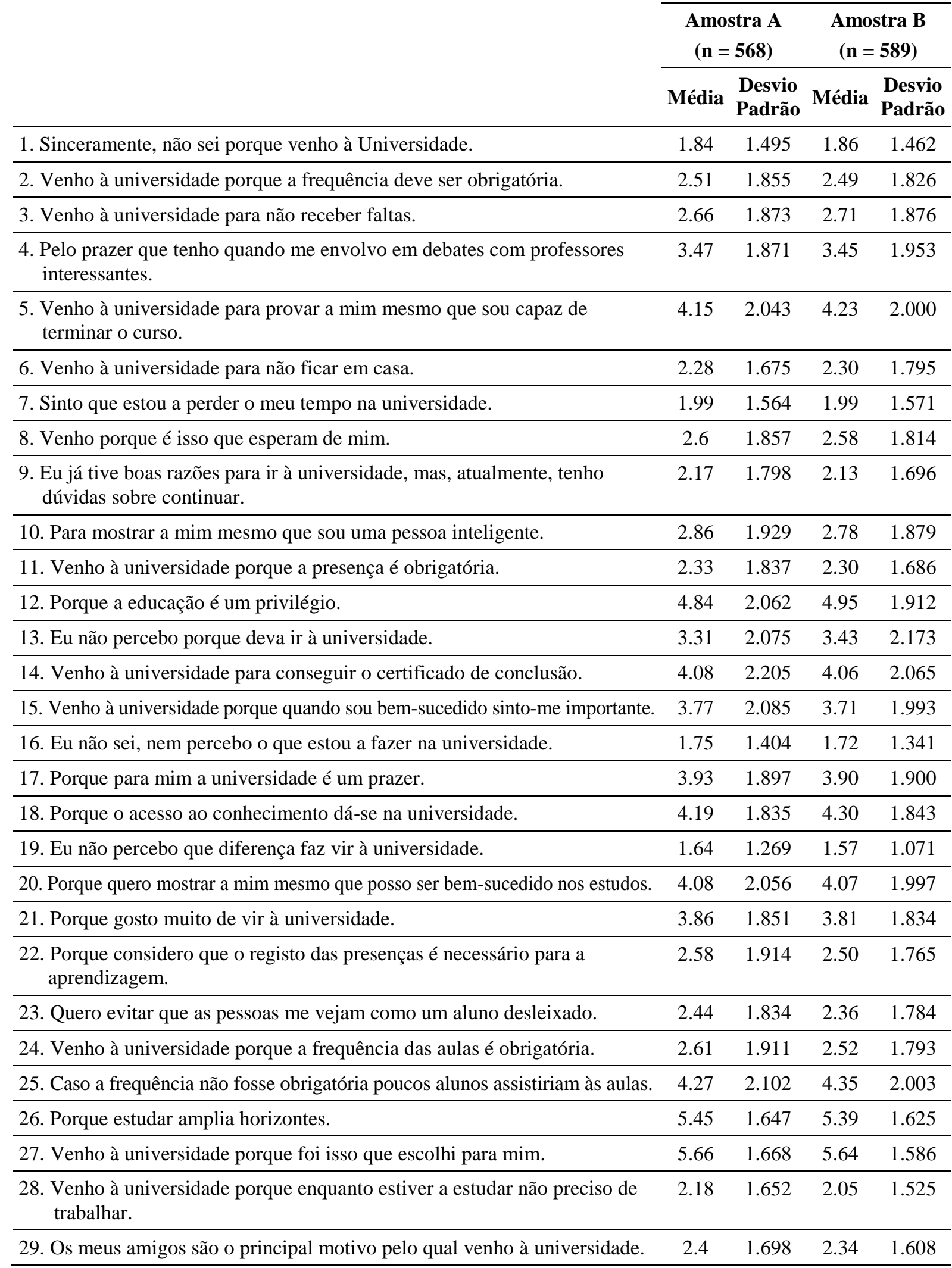

Nota. Os valores indicados reportam-se à escala de medida: 1-Nenhuma Correspondência; 2-Muito Pouca Correspondência; 3-Pouca Correspondência; 4-Alguma Correspondência; 5-Moderada Correspondência; 6-Muita Correspondência; 7-Total Correspondência. 
Verificamos que o item 27 apresentou a maior média em ambas as amostras (na Amostra A, M = 5.66; na Amostra $B, M=5.64$ ), seguido do item 26 (na Amostra $A, M=5.45$; na Amostra $B, M=5.39$ ). Verifica-se que, para ambas as amostras, o fato de estudar ampliar horizontes e a liberdade de escolha ao frequentar a universidade constituem elementos fulcrais na motivação académica das amostras. Por sua vez, o item 19 apresentou a média mais baixa em ambas as amostras (na Amostra A, M = 1.64; na Amostra $\mathrm{B}, \mathrm{M}=1.57$ ), pelo que, em ambas as amostras, a frequência do ensino superior é indispensável. Foi também observado, através do teste $t$ de Student, a não existência de diferenças significativas entre as amostras apresentadas.

Atendendo ao agrupamento dos itens anteriores nas dimensões que constituem a Escala de Motivação Académica (EMA), são apresentadas as estatísticas descritivas por cada dimensão na Tabela 2.

Tabela 2

Estatísticas: Dimensões da Escala de Motivação Académica

\begin{tabular}{llcccc} 
& & \multicolumn{2}{c}{$\begin{array}{c}\text { Amostra A } \\
\text { (n=568) }\end{array}$} & \multicolumn{2}{c}{$\begin{array}{c}\text { Amostra B } \\
\text { (n=589) }\end{array}$} \\
\hline Fator & Indicadores & Média & $\begin{array}{c}\text { Desvio } \\
\text { Padrão }\end{array}$ & $\begin{array}{c}\text { Média } \\
\text { Desvio } \\
\text { Padrão }\end{array}$ \\
\hline DESM: Desmotivação & $\begin{array}{l}\text { Q1; Q7, Q9, } \\
\text { Q13, Q16, Q19 }\end{array}$ & 2.12 & 1.04 & 2.12 & 0.99 \\
\hline MI: Motivação Intrínseca & Q4, Q17, Q21 & 3.75 & 1.61 & 3.72 & 1.61 \\
\hline $\begin{array}{l}\text { MERInte: Motivação Extrínseca por Regulação } \\
\text { Integrada }\end{array}$ & $\begin{array}{l}\text { Q12, Q18, Q26, } \\
\text { Q27 }\end{array}$ & 5.03 & 1.40 & 5.07 & 1.41 \\
\hline $\begin{array}{l}\text { MERIden: Motivação Extrínseca por Regulação } \\
\text { Identificada }\end{array}$ & $\begin{array}{l}\text { Q2, Q22, Q24, } \\
\text { Q25 }\end{array}$ & 2.99 & 1.36 & 2.97 & 1.24 \\
\hline $\begin{array}{l}\text { MERIntro: Motivação Extrínseca por Regulação } \\
\text { Introjetada }\end{array}$ & $\begin{array}{l}\text { Q5, Q8, Q10, } \\
\text { Q15, Q20, Q23 }\end{array}$ & 3.32 & 1.39 & 3.29 & 1.32 \\
\hline $\begin{array}{l}\text { MERExter: Motivação Extrínseca por Regulação } \\
\text { Externa }\end{array}$ & $\begin{array}{l}\text { Q3, Q6, Q11, } \\
\text { Q14, Q28, Q29 }\end{array}$ & 2.66 & 1.19 & 2.63 & 1.14 \\
\hline
\end{tabular}

Nota. Os valores indicados reportam-se à escala de medida: 1-Nenhuma Correspondência; 2-Muito Pouca Correspondência; 3-Pouca Correspondência; 4-Alguma Correspondência; 5-Moderada Correspondência; 6-Muita Correspondência; 7-Total Correspondência

Verificamos que a dimensão motivação extrínseca por regulação integrada apresentou a maior média em ambas as amostras (na Amostra $\mathrm{A}, \mathrm{M}=5.03$; na Amostra $\mathrm{B}, \mathrm{M}=5.07$ ), seguida da dimensão motivação intrínseca (na Amostra $\mathrm{A}, \mathrm{M}=3.75$; na Amostra $\mathrm{B}, \mathrm{M}=3.72$ ), pelo que, em ambas as amostras, embora o indivíduo revele gratificação e interesse e se identifique com o valor da atividade, esta não possui significado para si, pelo que age de acordo com os resultados que alcançará. Por sua vez, a dimensão desmotivação apresentou a menor média em ambas as amostras (na Amostra $\mathrm{A}, \mathrm{M}=2.12$; na Amostra B, M = 2.12), pelo que, ambas as amostras se apresentam motivadas.

\section{Análise fatorial confirmatória}

Tendo como base as seis dimensões propostas pela Teoria da Autodeterminação, bem como os itens da escala de avaliação que constitui cada dimensão, foi realizada uma análise de confiabilidade através da utilização do Alfa de Cronbach, isto é, foi realizada uma análise à consistência interna/fiabilidade dos diferentes itens que constituem as diferentes dimensões atendendo à Teoria da Autodeterminação. 
Tabela 3

Dimensões, Indicadores e Alfa de Cronbach para a Amostra A

\begin{tabular}{lcc}
\hline Fator & Indicadores & Alfa de Cronbach \\
\hline DESM: Desmotivação & Q1; Q7, Q9, Q13, Q16, Q19 & .734 \\
\hline MI: Motivação Intrínseca & Q4, Q17, Q21 & .825 \\
\hline MERInte: Motivação Extrínseca por Regulação Integrada & Q12, Q18, Q26, Q27 & .780 \\
\hline MERIden: Motivação Extrínseca por Regulação Identificada & Q2, Q22, Q24, Q25 & .650 \\
\hline MERIntro: Motivação Extrínseca por Regulação Introjetada & Q5, Q8, Q10, Q15, Q20, Q23 & .800 \\
\hline MERExter: Motivação Extrínseca por Regulação Externa & Q3, Q6, Q11, Q14, Q28, Q29 & .729 \\
\hline
\end{tabular}

No geral, excluindo a dimensão da Motivação Extrínseca por Regulação Identificada, todas as dimensões apresentam um nível de consistência interna aceitável, sendo que a Motivação Intrínseca apresentou o maior nível de consistência interna $(\alpha=.825)$, seguido da Motivação Extrínseca por Regulação Introjetada $(\alpha=.825)$, ambas apresentando um bom nível de fiabilidade.

Foi conduzida uma Análise Fatorial Confirmatória para testar um modelo composto por seis fatores correlacionados entre si: Desmotivação, Motivação Intrínseca, Motivação Extrínseca por Regulação Integrada, Motivação Extrínseca por Regulação Identificada, Motivação Extrínseca por Regulação Introjetada e Motivação Extrínseca por Regulação Externa na Amostra A. Os fatores Desmotivação, Motivação Extrínseca por Regulação Introjetada e Motivação Extrínseca por Regulação Externa são compostos por seis indicadores, enquanto que os fatores Motivação Intrínseca e Motivação Extrínseca por Regulação Integrada são compostos por três indicadores e a Motivação Extrínseca por Regulação Identificada é composta por quatro indicadores, tal como descrito na Tabela 2, apresentada anteriormente. Como forma de estimar os parâmetros de cada item, para dar escala aos fatores, fixou-se a variância destes em 1 .

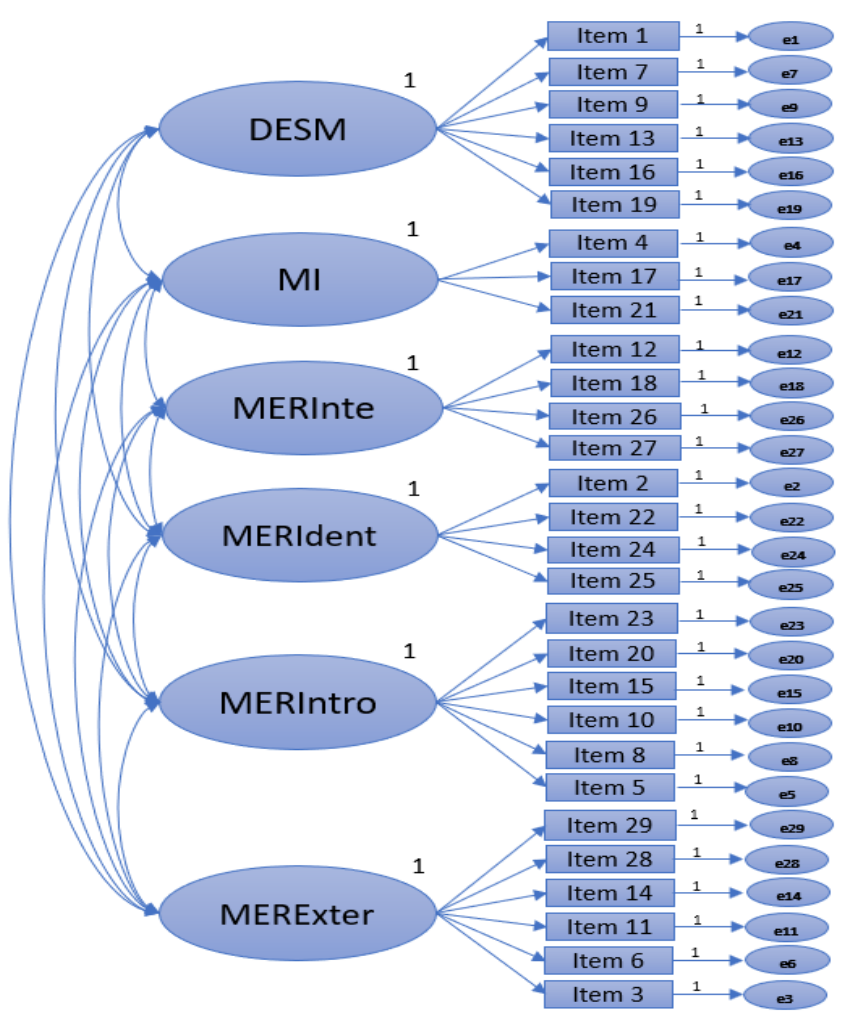

Figura 1. Modelo de 6 Fatores Correlacionados Entre Si Fonte: Elaborada pelos autores. 
Neste sentido, foram conduzidas analises preliminares com o objetivo de verificar a distribuição de todas as variáveis manifestas. A existência de outliers foi avaliada pela distância quadrada de Mahalanobis $\left(D^{2}\right)$ e pela referência dos valores de $p 1$ e $p 2$ (Kline, 2005; Marôco, 2010), tendo-se definido como outliers $p 1$ e $p 2$ inferiores a .001 e simultaneamente $D^{2}$ inferior a 20 . Neste sentido, não se verificou a existência de outliers na amostra, dado que foram apresentados valores máximos de .064 para $p 1, .000$ para $p 2$ e 87.624 para $D^{2}$. Deve ter-se em atenção que, apesar da violação do pressuposto da continuidade e normalidade multivariada associado à escala do tipo Likert, vários estudos de simulação computacional demostram que os resultados obtidos são credíveis desde que o número de classes da variável seja elevado (pelo menos cinco, neste caso constituída por sete classes, tal como descrito anteriormente) e a distribuição das frequências das classes se aproxime da distribuição normal, o que se verifica (Kline, 2005; Marôco, 2010). A normalidade univariada foi avaliada pelos coeficientes de assimetria $(S k<$ I3I) e de curtose $(K u<$ I10I) (Kline, 2005; Marôco, 2010) não se identificando desvios grandes à normalidade, dado que se verificou um valor máximo de I2.397I para a assimetria e de I5.661I para a curtose.

Para medir a qualidade do ajustamento, utilizam-se os valores de referência recomendados por Arbuckle e Wothke (2003), Luque (2000) e Karjaluoto, Mattila e Pento (2002), de acordo com a Tabela 4.

Tabela 4

Valores de Referência para a Avaliação do Ajustamento dos Modelos de Equações Estruturais

\begin{tabular}{|c|c|c|}
\hline Índices de ajustamento & Descrição & Valores recomendados \\
\hline $\begin{array}{l}\text { Índice absoluto: } \\
\text { Qui-quadrado/g. } 1 . \\
\mathrm{X}^{2} \text { /g.l. }\end{array}$ & $\begin{array}{l}\text { Cálculo da diferença entre as } \\
\text { matrizes de covariância observadas e } \\
\text { esperadas; ajustado aos graus de } \\
\text { liberdade }\end{array}$ & $\begin{array}{l}=1 \text { ajustamento muito bom. } \\
<2 \text { bom ajustamento. } \\
<5 \text { ajustamento aceitável. } \\
>5 \text { ajustamento inaceitável. }\end{array}$ \\
\hline $\begin{array}{l}\text { Índice de discrepância: } \\
\text { RMSEA (Root mean square error of } \\
\text { approximation) }\end{array}$ & $\begin{array}{l}\text { Indica o ajustamento global do } \\
\text { modelo relativamente aos graus de } \\
\text { liberdade }\end{array}$ & $\begin{array}{l}<0,05 \text { ajustamento perfeito; } \\
<0,08 \text { ajustamento aceitável }\end{array}$ \\
\hline $\begin{array}{l}\text { Índice relativo: } \\
\text { NFI (Normed of fit index) }\end{array}$ & $\begin{array}{l}\text { Compara o modelo proposto com o } \\
\text { modelo nulo; não ajustado aos graus } \\
\text { de liberdade }\end{array}$ & $\begin{array}{l}\text { De } 0 \text { (sem ajustamento) a } 1 \\
\text { (ajustamento perfeito); } \\
>0,80 \text { ajustamento recomendado }\end{array}$ \\
\hline $\begin{array}{l}\text { Índice relativo: } \\
\text { CFI (Comparative fit index) }\end{array}$ & $\begin{array}{l}\text { Índice de adequação global do } \\
\text { modelo }\end{array}$ & $\begin{array}{l}\text { De } 0 \text { (sem ajustamento) a } 1 \\
\text { (ajustamento perfeito); } \\
0,80-0,90 \text { ajustamento aceitável } \\
>0,90 \text { ajustamento recomendado }\end{array}$ \\
\hline
\end{tabular}

Nota. Fontes: Arbuckle, J., \& Wothke W. (2003). AMOS 5.0: User's guide. Chicago, IL: Smallwaters Corporation.; Luque, T. (2000). Técnicas de análisis de datos en investigación de mercados. Madrid: Ediciones Pirámide; Karjaluoto, H., Mattila, M., \& Pento, T. (2002). Factors underlying attitude formation towards online banking in Finland. International Journal of Bank Marketing, 20(6), 261-272. Recuperado de. https://www.emeraldinsight.com/doi/abs/10.1108/02652320210446724. https://doi.org/10.1108/02652320210446724

Os índices de ajustamento utilizados são justificados por Kline (2005) que sugere testes que incluem o RMSEA, CFI, o qui-quadrado do modelo, os seus graus de liberdade e o valor de prova $p$. Thompson (2000, pp. 270-271) recomenda o "Comparative Fit Index (CFI)" e a "Root Mean Square Error of Approximation (RMSEA)" como sendo os mais utilizados para a avaliação do ajustamento dos modelos. McDonald e Ho (2002), baseados na revisão da literatura, consideram que os modelos de medida do ajustamento mais utilizados são CFI, GFI, NFI e NNFI. Hooper, Coughlan e Mullen (2008) sugerem a utilização do qui-quadrado do modelo, os seus graus de liberdade e o valor de prova $p$, RMSEA, CFI e o NFI. Esta seleção é baseada na revisão da literatura e no facto de que estes índices são menos sensíveis à dimensão da amostra, má especificação do modelo e estimativas dos parâmetros. 
Na análise foi utilizado como estimador a Máxima Verossimilhança. Os resultados mostram um ajustamento razoável do modelo, $X^{2} / d f=4.999, \mathrm{CFI}=.818, \mathrm{NFI}=.783, \mathrm{RMSEA}=.084,90 \% \mathrm{CI}[.080$, .088 ], $P$ (rmsea < $=.05)<.001$. O fator Motivação Extrínseca por Regulação Identificada foi retirado do modelo, uma vez que os indicadores correspondentes não representam adequadamente a variável latente (Guimarães \& Bzuneck, 2008; Leal et al., 2011) apresentando o menor Alfa de Cronbach (.622). Por outro lado, a eliminação deste fator resulta também numa diminuição do AIC, sendo benéfico para o modelo (com o fator Motivação Extrínseca por Regulação Identificada, AIC = 1955.517, sem o fator, AIC = 1568.262). Logo, fez-se uma análise dos resíduos estandardizados e índices de modificação (IM), com o objetivo de identificar possíveis fontes locais desse resultado.

Constatou-se que o item 8 apresentou índices de modificação elevados (superior entre o Item 7 e Item $8, \mathrm{IM}=165.22$ ) e resíduos estandardizados igualmente elevados (verificou-se um resíduo superior de 10.650 entre o item 8 e item 16). Para além disso, verificou-se ainda que ao retirar o item 8 o Alfa de Cronbach aumenta para .821. Neste sentido, o item 8 foi retirado do modelo. Constatou-se igualmente que o item 14 apresentou índices de modificação elevados (superior entre o item 15 e item 14, IM = 138.468) e resíduos estandardizados elevados (verificou-se um resíduo superior de 10.396 entre o item 15 e 14). Para além disso, verificou-se ainda que com a retirada do item 14 o Alfa de Cronbach aumenta para .803. Atendendo a ter sido verificado índices de modificação também elevados entre os itens 28 e 29 (Item $28 \rightarrow$ Item 29, IM = 84.589; e Item $29 \rightarrow$ Item 28, IM = 79.261) e resíduos elevados (8.476), estes itens foram correlacionados. Verificou-se ainda, para o item 6 , igualmente índices de modificação elevados (superior entre os itens 28 e $6, \mathrm{IM}=47.140$, seguido dos itens 10 e $6, \mathrm{IM}=41.606$ ). Para além disso, este item apresentou também resíduos elevados, tendo sido verificado um resíduo superior de 6.345 entre os itens 23 e 6 . Neste sentido, o item 6 foi igualmente retirado do modelo.

$\mathrm{O}$ modelo reespecificado mostrou um bom ajustamento, $X^{2} / g l=3.322, \mathrm{CFI}=.920, \mathrm{NFI}=.890$, RMSEA $=.064,90 \%$ CI [.056, .067], $P($ rmsea $<=.05)<.001$. A análise das estimativas dos parâmetros revelou que os fatores MERInte e MI apresentaram a maior correlação, estando fortemente correlacionados $(\mathrm{r}=.817, \mathrm{p}<.001)$, seguindo-se os fatores MERInte e MERIntro $(r=.586, p<.001) \mathrm{e}$ os fatores DESM e MERExter $(r=.529, p<.001)$. Todos os índices saturam no respetivo fator com pesos fatoriais a variar entre .155 e .932 .

Tabela 5

\section{Parâmetros Estimados para o Modelo Final na Amostra A}

\begin{tabular}{lcccc}
\hline Paths & $\boldsymbol{B}$ & $\boldsymbol{S E}$ & $\boldsymbol{p}$ & $\boldsymbol{b}$ \\
\hline Desmotivação $\rightarrow$ Item 1 & 1.101 & .056 & $<.001$ & .737 \\
\hline Desmotivação $\rightarrow$ Item 7 & 1.259 & 0.57 & $<.001$ & .806 \\
\hline Desmotivação $\rightarrow$ Item 9 & 1.360 & .067 & $<.001$ & .757 \\
\hline Desmotivação $\rightarrow$ Item 13 & 0.321 & .092 & $<.001$ & .155 \\
\hline Desmotivação $\rightarrow$ Item 16 & 1.166 & .050 & $<.001$ & .831 \\
\hline Desmotivação $\rightarrow$ Item 19 & 0.891 & .048 & $<.001$ & .703 \\
\hline Motivação Extrínseca por Regulação Integrada $\rightarrow$ Item 12 & 1.268 & .083 & $<.001$ & .616 \\
\hline Motivação Extrínseca por Regulação Integrada $\rightarrow$ Item 18 & 1.347 & .070 & $<.001$ & .735 \\
\hline Motivação Extrínseca por Regulação Integrada $\rightarrow$ Item 26 & 1.238 & .063 & $<.001$ & .752 \\
\hline Motivação Extrínseca por Regulação Integrada $\rightarrow$ Item 27 & 1.129 & .066 & $<.001$ & .678 \\
\hline Motivação Intrínseca $\rightarrow$ Item 4 & 1.043 & .075 & $<.001$ & .558 \\
\hline Motivação Intrínseca $\rightarrow$ Item 17 & 1.767 & .063 & $<.001$ & .932 \\
\hline
\end{tabular}




\section{Tabela 5 (continuação)}

\begin{tabular}{|c|c|c|c|c|}
\hline Paths & $\boldsymbol{B}$ & $S E$ & $p$ & $b$ \\
\hline Motivação Intrínseca $\rightarrow$ Item 21 & 1.626 & .063 & $<.001$ & .879 \\
\hline Motivação Extrínseca por Regulação Introjetada $\rightarrow$ Item 23 & 0.977 & .077 & $<.001$ & .553 \\
\hline Motivação Extrínseca por Regulação Introjetada $\rightarrow$ Item 20 & 1.726 & .075 & $<.001$ & .840 \\
\hline Motivação Extrínseca por Regulação Introjetada $\rightarrow$ Item 15 & 1.443 & .082 & $<.001$ & .693 \\
\hline Motivação Extrínseca por Regulação Introjetada $\rightarrow$ Item 10 & 1.387 & .075 & $<.001$ & .720 \\
\hline Motivação Extrínseca por Regulação Introjetada $\rightarrow$ Item 5 & 1.378 & .081 & $<.001$ & .675 \\
\hline Motivação Extrínseca por Regulação Externa $\rightarrow$ Item 29 & 0.555 & .073 & $<.001$ & .327 \\
\hline Motivação Extrínseca por Regulação Externa $\rightarrow$ Item 28 & 0.394 & .072 & $<.001$ & .239 \\
\hline Motivação Extrínseca por Regulação Externa $\rightarrow$ Item 11 & 1.656 & .069 & $<.001$ & .903 \\
\hline Motivação Extrínseca por Regulação Externa $\rightarrow$ Item 3 & 1.646 & .071 & $<.001$ & .879 \\
\hline \multicolumn{5}{|l|}{ Desmotivação } \\
\hline Motivação Extrínseca por Regulação Externa & 0.529 & .036 & $<.001$ & .529 \\
\hline Motivação Extrínseca por Regulação Introjetada & 0.002 & .048 & .917 & .002 \\
\hline Motivação Extrínseca por Regulação Integrada & -0.349 & .045 & $<.001$ & -.349 \\
\hline Motivação Intrínseca & -0.442 & .039 & $<.001$ & -.442 \\
\hline \multicolumn{5}{|l|}{ Motivação Intrínseca } \\
\hline Motivação Extrínseca por Regulação Externa & -0.219 & .045 & $<.001$ & -.219 \\
\hline Motivação Extrínseca por Regulação Introjetada & 0.343 & .043 & $<.001$ & .343 \\
\hline \multicolumn{5}{|l|}{ Motivação Extrínseca por Regulação Integrada } \\
\hline Motivação Extrínseca por Regulação Externa & -0.052 & .050 & .298 & -.052 \\
\hline Motivação Extrínseca por Regulação Introjetada & 0.586 & .037 & $<.001$ & .586 \\
\hline Motivação Intrínseca & 0.817 & .023 & $<.001$ & .817 \\
\hline \multicolumn{5}{|l|}{ Motivação Extrínseca por Regulação Introjetada } \\
\hline Motivação Extrínseca por Regulação Externa & 0.222 & .046 & $<.001$ & .222 \\
\hline \multicolumn{5}{|l|}{ Item 29} \\
\hline Item 28 & 1.060 & .118 & $<.001$ & .412 \\
\hline
\end{tabular}

Nota. Legenda: B - Saturação não estandardizada; SE - Erro padrão, p - valor de prova; b - Saturação estandardizada.

Considerando agora a Amostra B, tendo como base as dimensões consideradas anteriormente, foi realizada uma análise de confiabilidade através da utilização do Alfa de Cronbach, isto é, foi realizada uma análise à consistência interna/fiabilidade. 
Tabela 6

Dimensões, Indicadores e Alfa de Cronbach para a Amostra B

\begin{tabular}{lcc}
\hline Fator & Indicadores & Alfa de Cronbach \\
\hline DESM: Desmotivação & Q1; Q7, Q9, Q13, Q16, Q19 & .745 \\
\hline MI: Motivação Intrínseca & Q4, Q17, Q21 & .810 \\
\hline MERInte: Motivação Extrínseca por Regulação Integrada & Q12, Q18, Q26, Q27 & .823 \\
\hline MERIntro: Motivação Extrínseca por Regulação Introjetada & Q5, Q10, Q15, Q20, Q23 & .731 \\
\hline MERExter: Motivação Extrínseca por Regulação Externa & Q3, Q11, Q28, Q29 & .694 \\
\hline
\end{tabular}

No geral, todas as dimensões apresentam um nível de consistência interna aceitável, sendo que a Motivação Extrínseca por Regulação Integrada apresentou o maior nível de consistência interna $(\alpha=$ $.823)$, seguido da Motivação Intrínseca $(\alpha=.825)$, ambas apresentando um bom nível de fiabilidade.

Foi conduzida novamente uma Análise Fatorial Confirmatória para testar o modelo proposto anteriormente numa nova amostra, a Amostra B, como forma de validação do modelo.

Neste sentido, foram conduzidas análises preliminares com o objetivo de verificar a distribuição de todas as variáveis manifestas. A existência de outliers foi avaliada pela distância quadrada de Mahalanobis $\left(D^{2}\right)$ e pela referência dos valores de $p 1$ e $p 2$, tendo-se definido como outliers $p 1$ e $p 2$ inferiores a .001 e simultaneamente $D^{2}$ inferior a 20, tal como foi definido no anterior modelo. Neste sentido, não se verificou a existência de outliers na amostra, dado que foram apresentados valores máximos de .077 para $p 1, .000$ para $p 2$ e 32.023 para $D^{2 .}$ A normalidade univariada foi avaliada pelos coeficientes de assimetria $(S k<|3|)$ e de curtose $(K u<|10|)$ não se identificando desvios grandes à normalidade, dado que se verificou um valor máximo de |2.439| para a assimetria e de |6.732| para a curtose. Na análise foi utilizado como estimador a Máxima Verossimilhança. Os resultados mostram um bom ajustamento do modelo, $X^{2} / \mathrm{gl}=3.204, \mathrm{CFI}=.922, \mathrm{RMSEA}=.061,90 \%$ CI $[.056, .067], P$ $($ rmsea $<=.05)<.001$.

A análise das estimativas dos parâmetros revelou que os fatores MERInte e MI apresentaram a maior correlação, estando fortemente correlacionados $(r=.789, p<.001)$, seguindo-se os fatores MERInte e MERIntro $(r=.502, p<.001)$ e os fatores DESM e MERExter $(r=.452, p<.001)$. Todos os índices saturam no respetivo fator com pesos fatoriais a variar entre $.119 \mathrm{e} .912$.

Tabela 7

Parâmetros Estimados para o Modelo Final na Amostra B

\begin{tabular}{lcccc}
\hline Paths & $\boldsymbol{B}$ & $\boldsymbol{S E}$ & $\boldsymbol{p}$ & $\boldsymbol{b}$ \\
\hline Desmotivação $\rightarrow$ Item 1 & .899 & .057 & $<.001$ & .615 \\
\hline Desmotivação $\rightarrow$ Item 7 & 1.324 & .055 & $<.001$ & .843 \\
\hline Desmotivação $\rightarrow$ Item 9 & 1.307 & .062 & $<.001$ & .771 \\
\hline Desmotivação $\rightarrow$ Item 13 & 0.258 & .095 & $<.001$ & .119 \\
\hline Desmotivação $\rightarrow$ Item 16 & 1.087 & .048 & $<.001$ & .811 \\
\hline Desmotivação $\rightarrow$ Item 19 & 0.710 & .041 & $<.001$ & .663 \\
\hline Motivação Extrínseca por Regulação Integrada $\rightarrow$ Item 12 & 1.235 & .074 & $<.001$ & .646 \\
\hline Motivação Extrínseca por Regulação Integrada $\rightarrow$ Item 18 & 1.386 & .068 & $<.001$ & .753 \\
\hline
\end{tabular}




\section{Tabela 7 (continuação)}

\begin{tabular}{|c|c|c|c|c|}
\hline Paths & $\boldsymbol{B}$ & $S E$ & $p$ & $\boldsymbol{b}$ \\
\hline Motivação Extrínseca por Regulação Integrada $\rightarrow$ Item 26 & 1.342 & .057 & $<.001$ & .826 \\
\hline Motivação Extrínseca por Regulação Integrada $\rightarrow$ Item 27 & 1.198 & .058 & $<.001$ & .756 \\
\hline Motivação Intrínseca $\rightarrow$ Item 4 & 1.056 & .078 & $<.001$ & .541 \\
\hline Motivação Intrínseca $\rightarrow$ Item 17 & 1.723 & .063 & $<.001$ & .908 \\
\hline Motivação Intrínseca $\rightarrow$ Item 21 & 1.631 & .062 & $<.001$ & .890 \\
\hline Motivação Extrínseca por Regulação Introjetada $\rightarrow$ Item 23 & 0.728 & .076 & $<.001$ & .439 \\
\hline Motivação Extrínseca por Regulação Introjetada $\rightarrow$ Item 20 & 1.654 & .074 & $<.001$ & .829 \\
\hline Motivação Extrínseca por Regulação Introjetada $\rightarrow$ Item 15 & 1.300 & .079 & $<.001$ & .653 \\
\hline Motivação Extrínseca por Regulação Introjetada $\rightarrow$ Item 10 & 1.277 & .074 & $<.001$ & 680 \\
\hline Motivação Extrínseca por Regulação Introjetada $\rightarrow$ Item 5 & 1.368 & .078 & $<.001$ & .685 \\
\hline Motivação Extrínseca por Regulação Externa $\rightarrow$ Item 29 & 0.566 & .069 & $<.001$ & .352 \\
\hline Motivação Extrínseca por Regulação Externa $\rightarrow$ Item 28 & 0.412 & .066 & $<.001$ & .270 \\
\hline Motivação Extrínseca por Regulação Externa $\rightarrow$ Item 11 & 1.362 & .067 & $<.001$ & .809 \\
\hline Motivação Extrínseca por Regulação Externa $\rightarrow$ Item 3 & 1.710 & .074 & $<.001$ & .912 \\
\hline \multicolumn{5}{|l|}{ Desmotivação } \\
\hline Motivação Extrínseca por Regulação Externa & 0.452 & .039 & $<.001$ & .452 \\
\hline Motivação Extrínseca por Regulação Introjetada & 0.064 & .048 & .186 & .064 \\
\hline Motivação Extrínseca por Regulação Integrada & -0.386 & .042 & $<.001$ & -.386 \\
\hline Motivação Intrínseca & -0.484 & .037 & $<.001$ & -.484 \\
\hline \multicolumn{5}{|l|}{ Motivação Intrínseca } \\
\hline Motivação Extrínseca por Regulação Externa & -0.198 & .045 & $<.001$ & -.198 \\
\hline Motivação Extrínseca por Regulação Introjetada & 0.284 & .044 & $<.001$ & .284 \\
\hline \multicolumn{5}{|l|}{ Motivação Extrínseca por Regulação Integrada } \\
\hline Motivação Extrínseca por Regulação Externa & -0.094 & .048 & .048 & -.094 \\
\hline Motivação Extrínseca por Regulação Introjetada & 0.502 & .039 & $<.001$ & .502 \\
\hline Motivação Intrínseca & 0.789 & .022 & $<.001$ & .789 \\
\hline \multicolumn{5}{|l|}{ Motivação Extrínseca por Regulação Introjetada } \\
\hline Motivação Extrínseca por Regulação Externa & 0.271 & .045 & $<.001$ & .271 \\
\hline \multicolumn{5}{|l|}{ Item 29} \\
\hline Item 28 & 0.661 & .097 & $<.001$ & .300 \\
\hline
\end{tabular}

Nota. Legenda: B - Saturação não estandardizada; SE - Erro padrão, p - valor de prova; b - Saturação estandardizada.

\section{Análise de grupos múltiplos}

Com o objetivo de testar a invariância do modelo proposto anteriormente entre a Amostra A e a Amostra B, foi conduzida uma análise de grupos múltiplos. Como forma de estabelecer a escala das variáveis latentes foi mantida a variância destes fixa em 1. 
Foi conduzida uma primeira análise com todos os parâmetros livres. Os resultados mostraram um bom ajustamento do modelo, $X^{2}=(15, N=1157)=1292.109, p<.001, \mathrm{CFI}=.921$, RMSEA $=.044$, $P($ rmsea $\leq .05)=1.000$.

O teste das diferenças do qui quadrado revelou que o modelo de medida não era invariante entre as amostras, $X^{2}(22)=37.743, p=.020$, sendo as diferenças apresentadas na Tabela 8 .

Tabela 8

Diferenças Estimadas Entre os Parâmetros Estimados para o Modelo Final

\begin{tabular}{|c|c|c|c|c|c|}
\hline & & & & \\
\hline & & $B$ & $S E$ & $p$ & \\
\hline \multirow[t]{2}{*}{ DESM $\rightarrow$ Item 1} & Amostra A & 1.101 & .056 & $<.001$ & Diferente \\
\hline & Amostra B & 0.899 & .057 & $<.001$ & \\
\hline \multirow[t]{2}{*}{ DESM $\rightarrow$ Item 7} & Amostra A & 1.259 & .057 & $<.001$ & Igual \\
\hline & Amostra B & 1.324 & .055 & $<.001$ & \\
\hline \multirow[t]{2}{*}{ DESM $\rightarrow$ Item 9} & Amostra A & 1.360 & .067 & $<.001$ & Igual \\
\hline & Amostra B & 1.307 & .062 & $<.001$ & \\
\hline \multirow[t]{2}{*}{ DESM $\rightarrow$ Item 13} & Amostra A & 0.321 & .092 & $<.001$ & Igual \\
\hline & Amostra B & 0.258 & .095 & $<.001$ & \\
\hline \multirow[t]{2}{*}{ DESM $\rightarrow$ Item 16} & Amostra A & 1.166 & .050 & $<.001$ & Igual \\
\hline & Amostra B & 1.087 & .048 & $<.001$ & \\
\hline \multirow[t]{2}{*}{$\mathrm{DESM} \rightarrow$ Item 19} & Amostra A & 0.891 & .048 & $<.001$ & Diferente \\
\hline & Amostra B & 0.71 & .041 & $<.001$ & \\
\hline \multirow[t]{2}{*}{ MERInte $\rightarrow$ Item 12} & Amostra A & 1.268 & .083 & $<.001$ & Igual \\
\hline & Amostra B & 1.235 & .074 & $<.001$ & \\
\hline \multirow[t]{2}{*}{ MERInte $\rightarrow$ Item 18} & Amostra A & 1.347 & .070 & $<.001$ & Igual \\
\hline & Amostra B & 1.386 & .068 & $<.001$ & \\
\hline \multirow[t]{2}{*}{ MERInte $\rightarrow$ Item 26} & Amostra A & 1.238 & .063 & $<.001$ & Igual \\
\hline & Amostra B & 1.342 & .057 & $<.001$ & \\
\hline \multirow[t]{2}{*}{ MERInte $\rightarrow$ Item 27} & Amostra A & 1.129 & .066 & $<.001$ & Igual \\
\hline & Amostra B & 1.198 & .058 & $<.001$ & \\
\hline \multirow[t]{2}{*}{ MI $\rightarrow$ Item 4} & Amostra A & 1.043 & .075 & $<.001$ & Igual \\
\hline & Amostra B & 1.056 & .078 & $<.001$ & \\
\hline \multirow[t]{2}{*}{ MI $\rightarrow$ Item 17} & Amostra A & 1.767 & .063 & $<.001$ & Igual \\
\hline & Amostra B & 1.723 & .063 & $<.001$ & \\
\hline \multirow[t]{2}{*}{ MI $\rightarrow$ Item 21} & Amostra A & 1.626 & .063 & $<.001$ & Igual \\
\hline & Amostra B & 1.631 & .062 & $<.001$ & \\
\hline \multirow[t]{2}{*}{ MERIntro $\rightarrow$ Item 23} & Amostra A & 0.977 & .077 & $<.001$ & Igual \\
\hline & Amostra B & 0.782 & .076 & $<.001$ & \\
\hline
\end{tabular}




\section{Tabela 8 (continuação)}

\begin{tabular}{|c|c|c|c|c|c|}
\hline & & & \\
\hline & & $\boldsymbol{B}$ & $S E$ & $p$ & \\
\hline \multirow[t]{2}{*}{ MERIntro $\rightarrow$ Item 20} & Amostra A & 1.726 & .075 & $<.001$ & \multirow[t]{2}{*}{ Igual } \\
\hline & Amostra B & 1.654 & .074 & $<.001$ & \\
\hline \multirow[t]{2}{*}{ MERIntro $\rightarrow$ Item 15} & Amostra A & 1.443 & .082 & $<.001$ & \multirow[t]{2}{*}{ Igual } \\
\hline & Amostra B & 1.300 & .079 & $<.001$ & \\
\hline \multirow[t]{2}{*}{ MERIntro $\rightarrow$ Item 10} & Amostra A & 1.387 & .075 & $<.001$ & \multirow[t]{2}{*}{ Igual } \\
\hline & Amostra B & 1.277 & .074 & $<.001$ & \\
\hline \multirow[t]{2}{*}{ MERIntro $\rightarrow$ Item 5} & Amostra A & 1.378 & .081 & $<.001$ & \multirow[t]{2}{*}{ Igual } \\
\hline & Amostra B & 1.368 & .078 & $<.001$ & \\
\hline \multirow[t]{2}{*}{ MERExter $\rightarrow$ Item 29} & Amostra A & 0.555 & .073 & $<.001$ & \multirow[t]{2}{*}{ Igual } \\
\hline & Amostra B & 0.566 & .069 & $<.001$ & \\
\hline \multirow[t]{2}{*}{ MERExter $\rightarrow$ Item 28} & Amostra A & 0.394 & .072 & $<.001$ & \multirow[t]{2}{*}{ Igual } \\
\hline & Amostra B & 0.412 & .066 & $<.001$ & \\
\hline \multirow[t]{2}{*}{ MERExter $\rightarrow$ Item 11} & Amostra A & 1.656 & .069 & $<.001$ & \multirow[t]{2}{*}{ Diferente } \\
\hline & Amostra B & 1.362 & .067 & $<.001$ & \\
\hline \multirow[t]{2}{*}{ MERExter $\rightarrow$ Item 3} & Amostra A & 1.646 & .071 & $<.001$ & \multirow[t]{2}{*}{ Igual } \\
\hline & Amostra B & 1.710 & .074 & $<.001$ & \\
\hline
\end{tabular}

Nota. Legenda: B - Saturação não estandardizada; SE - Erro padrão, p - valor de prova.

Com base na análise anterior os pesos fatoriais fixos dos itens 1, 19 e 11 foram libertados. Verificou-se que o modelo de correlação fixa é invariante entre ambas as amostras, $X^{2}(29)=22.981, p$ $=.777$.

\section{Discussão de Resultados}

A análise fatorial confirmatória revelou que os indicadores da variável latente Motivação Extrínseca por Regulação Identificada não explicam adequadamente a respetiva variável apresentando o menor Alfa de Cronbach, sendo que tais evidências são constatadas na literatura. Nas análises levadas a cabo por, Guimarães e Bzuneck (2008), Leal et al. (2013), Rodrigues e Joly (2011) e Souza e Miranda (2017), o fator Motivação Extrínseca por Regulação Identificada foi igualmente eliminado da análise, dado não apresentar uma consistência interna aceitável e os respetivos indicadores também não apresentam uma carga fatorial aceitável ou foram identificados noutros fatores. Também na escala original elaborada por Vallerand et al. (1989) e traduzida por Sobral (2003) apresentou uma baixa consistência interna relativamente ao fator Motivação Extrínseca por Regulação Identificada (Bizarria, Carneiro, Silva, \& Tassigny, 2016; Viana \& Viana, 2012).

Avaliando os indicadores de ajustamento de outros estudos semelhantes (Davoglio et al., 2016; Núñez, Juan, Albo, Izquierdo, \& Gregorio, 2005, 2006; Smith, Davy, \& Rosenberg, 2010; Viana, 2012), verifica-se que o presente estudo apresenta um ajustamento idêntico aos restantes. A dimensão da amostra revelou-se ajustada atendendo à dimensão do país em estudo comparativamente com os restantes estudos apresentados na Tabela 5. Tendo em conta a Amostra B, esta apresenta o menor $X^{2}$ comparativamente com os restantes estudos e o segundo menor RMSEA. Por último, relativamente ao CFI este está alinhado com os restantes estudos. 
Tabela 9

Ajustamento de Análises Fatoriais Confirmatórias

\begin{tabular}{lcccccc}
\hline & & $\mathbf{N}$ & $\boldsymbol{X}^{\mathbf{2}}$ & $\boldsymbol{d f}$ & \multicolumn{1}{c}{ CFI } & RMSEA \\
\hline Presente Estudo Amostra A & Portugal & 586 & 658 & 198 & .92 & .064 \\
\hline Presente Estudo Amostra B & Portugal & 589 & 634 & 198 & .922 & .061 \\
\hline Davoglio et al. (2016) & Brasil & 715 & - & - & .93 & .07 \\
\hline Núñez, Juan, Albo, Izquierdo e Gregorio (2005) & Espanha & 636 & 884 & 320 & .93 & .06 \\
\hline Viana (2012) & Brasil & 278 & 646 & 321 & .905 & .06 \\
\hline Núñez, Juan, Albo, Izquierdo e Gregorio (2006) & Paraguai & 411 & 626 & 325 & .93 & .05 \\
\hline Smith, Davy e Rosenberg (2010) & EUA & 2078 & 2235 & 329 & 0,935 & .052 \\
\hline
\end{tabular}

Nota. Legenda: $X^{2}$ - qui-quadrado, df - graus de liberdade, CFI - Comparative fit index, RMSEA - Root mean square error of approximation. Fonte: Elaborada pelos autores.

\section{Considerações Finais}

A motivação, como forma de preditor do comportamento humano, em específico, a motivação no ensino superior, tem vindo a ser destaque de diversas investigações (Davoglio et al., 2016; Leal et al., 2013; Núñez \& León, 2018; Ramos, 2013; Reboredo \& Monteiro, 2015), tendo como objetivo o estudo dos fatores que influenciam a motivação académica. A importância crescente desta temática de investigação deve-se, essencialmente, ao facto de que um aluno orientado para a aprendizagem e para a busca por novos conhecimentos e desafios, isto é, um aluno motivado, terá um desempenho superior quando comparado com um aluno desmotivado (Cardoso \& Bzuneck, 2004). Neste sentido, é importante a utilização de escalas robustas na avaliação da motivação a nível académico.

Qualquer instrumento de medida deve ser continuamente validado através da utilização de diferentes amostras e em momentos diferentes (Guimarães \& Bzuneck, 2008). Esta investigação teve como objetivo o estudo das propriedades psicométricas da Escala de Motivação Académica que levou a um aprimoramento teórico através da avaliação do contributo da medição do indicador para o respetivo fator e a sua posterior validação e teste de invariância. Os resultados mostram um bom ajustamento do modelo, para a amostra A $X^{2} / g l=3.322, \mathrm{CFI}=.920, \mathrm{NFI}=.890, \mathrm{RMSEA}=.064,90 \% \mathrm{CI}[.056, .067]$, $P($ rmsea $<=.05)<.001$ e para a amostra B $X^{2} / \mathrm{gl}=3.204, \mathrm{CFI}=.922, \mathrm{RMSEA}=.061,90 \% \mathrm{CI}[.056$, $.067], P($ rmsea $<=.05)<.001$. Por outro lado, verificou-se que as dimensões Motivação Extrínseca por Regulação Integrada e Motivação Intrínseca estava fortemente correlacionados (para a amostra A r = $.817, \mathrm{p}<.001$ e para a amostra B $r=.789, p<.001$ ), seguindo-se as dimensões Motivação Extrínseca por Regulação Integrada e Motivação Extrínseca por Regulação Introjetada (para a amostra A $r=.586$, $p<.001$ e para a amostra B $r=.502, p<.001)$ e as dimensões Desmotivação e Motivação Extrínseca por Regulação Externa (para a amostra A $r=.529, p<.001$ e para a amostra $\mathrm{B} r=.452, p<.001$ ). Conclui-se ainda que quando libertos os pesos fatoriais dos itens 1,19 e 11 o modelo de escala proposto é invariante entre ambas as amostras, $X^{2}(29)=22.981, p=.777$.

Conclui-se, assim, que o modelo de escala proposto constitui um instrumento robusto para a avaliação da motivação académica tendo em conta a Teoria da Autodeterminação, embora a dimensão Motivação Extrínseca por Regulação Identificada não tenha sido avaliada dado os respetivos indicadores não explicarem de forma adequada o fator. Como proposta de investigação futura é sugerida a construção de novos indicadores que expliquem de forma adequada o fator enunciado. 


\section{Referências}

Arbuckle, J., \& Wothke W. (2003). AMOS 5.0: User's guide. Chicago, IL: Smallwaters Corporation.

Bizarria, F. P. de A., Carneiro, T. C. J., Silva, M. A. da, \& Tassigny, M. M. (2016). Academic motivation scale: Validity in the context of education distance course in public administration. Revista Capital Científico - Eletrônica, 14(4). Recuperado de http://www.spell.org.br/documentos/ver/44030/escalade-motivacao-academica--validade-no-contexto-da-educacao-a-distancia-em-curso-deadministracao-publica-/i/pt-br. http://doi.org/10.5935/2177-4153.20160031

Boruchovitch, E. (2008). A motivação para aprender de estudantes em cursos de formação de professores. Educação, 31(1), 30-38. Recuperado de http://www.redalyc.org/html/848/84806405/

Cardoso, L. R., \& Bzuneck, J. A. (2004). Motivação no ensino superior: Metas de realização e estratégias de aprendizagem. Psicologia Escolar e Educacional, 8(2), 145-155. Recuperado de http://www.scielo.br/pdf/pee/v8n2/v8n2a03.pdf. 85572004000200003

Castonguay, A., \& Miquelon, P. (2018). Motivational profiles, accelerometer-derived physical activity, and acute diabetes-related symptoms in adults with type 2 diabetes. BMC Public Health, 18(1). https://doi.org/10.1186/s12889-018-5376-y

Cunha, S. M., \& Carrilho, D. M. (2005). O processo de adaptação ao ensino superior e o rendimento acadêmico. Psicologia Escolar e Educacional, 9(2), 215-224. Recuperado de http://pepsic.bvsalud.org/scielo.php?script=sci_arttext\&pid=S1413-85572005000200004

Davoglio, T. R., Santos, B. S., \& Lettnin, C. C. (2016). Validação da escala de motivação acadêmica em universitários brasileiros. Avaliação e Políticas Públicas em Educação, 24(92), 522-545. https://doi.org/10.1590/S0104-40362016000300002

Deci, E. L., \& Gagné, M. (2005). Self-determination theory and work motivation. Journal of Organization Behavior, 26(4), 331-362. https://doi.org/10.1002/job.322

Deci, E. L., \& Ryan, R. M. (1985). Intrinsic motivation and self-determination in human behavior. New York: Plenum Press.

Eccheli, S. D. (2008). A motivação como prevenção da indisciplina. Educar em Revista, (32), 199-213. Recuperado de http://www.redalyc.org/html/1550/155013363014/

Guimarães, S. É. R., \& Bzuneck, J. A. (2008). Propriedades psicométricas de um instrumento para avaliação da motivação de universitários. Ciências \& Cognição, 13(1), 101-113. Recuperado de http://cienciasecognicao.org/revista/index.php/cec/article/view/682

Gustafsson, H., Carlin, M., Podlog, L., Stenling, A., \& Lindwall, M. (2018). Motivational profiles and burnout in elite athletes: A person-centered approach. Psychology of Sport and Exercise, 35, 118 125. https://doi.org/10.1016/J.PSYCHSPORT.2017.11.009

Haerens, L., Vansteenkiste, M., Meester, A., Delrue, J., Tallir, I., Broek, G. V., \& Aelterman, N. (2018). Different combinations of perceived autonomy support and control: Identifying the most optimal motivating style. Physical Education and Sport Pedagogy, 23(1), 16-36. https://doi.org/10.1080/17408989.2017.1346070

Hooper, D., Coughlan, J., \& Mullen, M. R. (2008). Structural equation modelling: Guidelines for determining model. Electronic Journal of Business Research Methods, 6(1), 53-60. Recuprado de https://arrow.dit.ie/cgi/viewcontent.cgi?article=1001\&context=buschmanart 
Karjaluoto, H., Mattila, M., \& Pento, T. (2002). Factors underlying attitude formation towards online banking in Finland. International Journal of Bank Marketing, 20(6), 261-272. Recuperado de https://www.emeraldinsight.com/doi/abs/10.1108/02652320210446724. https://doi.org/10.1108/02652320210446724

Kline, R. B. (2005). Principles and practice of structural equation modeling. (2nd ed.). New York: Guilford Press.

Leal, E. A., Miranda, G. J., \& Carmo, C. R. S. (2013). Self-determination theory: An analysis of student motivation in an accounting degree program. Revista Contabilidade \& Finanças, 24(62), 162173. https://doi.org/10.1590/S1519-70772013000200007

Luque, T. (2000). Técnicas de análisis de datos en investigación de mercados. Madrid: Ediciones Pirámide.

Marôco, J. (2010). Análise de equações estruturais: Fundamentos teóricos, software \& aplicações. Pêro Pinheiro: ReportNumber.

Martinelli, S. C., \& Bartholomeu, D. (2007). Escala de motivação acadêmica: Uma medida de motivação extrínseca e intrínseca. Avaliação Psicológica, 6(1), 21-31. Recuperado de http://www.redalyc.org/html/3350/335027181004/

McDonald, R. P., \& Ho, M. H. (2002). Principles and practice in reporting structural equation analyses. Psychological Methods, 7(1), 64-82. $\quad$ Recuperado de https://www.ncbi.nlm.nih.gov/pubmed/11928891

Menard, P., Bott, G., \& Crossler, R. (2017). User motivations in protecting information security: Protection motivation theory versus self-determination theory. Journal of Management Information Systems, 34(4), 1203-1230. Recuperado de https://eds.b.ebscohost.com/eds/pdfviewer/pdfviewer?vid=2\&sid=1a6cc91c-b123-4a57-99a7a575769b9903\%40pdc-v-sessmgr01. https://doi.org/10.1080/07421222.2017.1394083

Núñez, A., Juan, L., Albo, L. M., Izquierdo, N., \& Gregorio, J. (2005). Validación de la versión española de la Échelle de Motivation en Éducation. Psicothema, 17(2), 344-349. Recuperado de http://www.redalyc.org/articulo.oa?id=72717225

Núñez, A., Juan, L., Albo, L. M., Izquierdo, N., \& Gregorio, J. (2006). Validación de la Escala de Motivación Educativa (EME) en Paraguay. Interamerican Journal of Psychology, 40(3), 391398. Recuperado de http://www.redalyc.org/pdf/284/28440314.pdf

Núñez, J. L., \& León, J. (2018). Testing the relationships between global, contextual, and situational motivation: A longitudinal study of the horizontal, top-down, and bottom-up effects. Revista de Psicodidáctica, 23(1), 9-16. https://doi.org/10.1016/J.PSICOE.2017.07.003

Olafsen, A. H., Deci, E. L., \& Halvari, H. (2005). Basic psychological needs and work motivation: A longitudinal test of directionality. Motivation and Emotion, 42(2), 178-189. https://doi.org/10.1007/s11031-017-9646-2

Ramos, I. S. V. (2013, março 9). Motivação académica dos alunos do ensino superior. Psicologia, 115. Recuperado de http://www.psicologia.pt/artigos/textos/A0677.pdf

Reboredo, A. R., \& Monteiro, V. (2015). Motivação académica: Suas relações com o autoconceito, género e desempenho académico. Diversidade e Educação: Desafios Atuais, 85, 85-102. Recuperado de http://repositorio.ispa.pt/bitstream/10400.12/5555/1/CIPE_15_85-102.pdf

Rigby, C. S., \& Ryan, R. M. (2018). Self-determination theory in human resource development: New directions and practical considerations. Advances in Developing Human Resources, 20(2), 133147. https://doi.org/10.1177/1523422318756954 
Rodrigues, M. C., \& Joly, A. (2011). Avaliação da escala de motivação acadêmica em estudantes paulistas: Propriedades psicométricas. Psico-USF, 16(2), 175-184. Recuperado de http://www.redalyc.org/pdf/4010/401041440006.pdf

Sebire, S. J., Toumpakari, Z., Turner, K. M., Cooper, A. R., Page, A. S., Malpass, A., \& Andrews, R. C. (2018). "I've made this my lifestyle now": A prospective qualitative study of motivation for lifestyle change among people with newly diagnosed type two diabetes mellitus. BMC Public Health, 18(1), 204. https://doi.org/10.1186/s12889-018-5114-5

Sheehan, R. B., Herring, M. P., \& Campbell, M. J. (2018). Associations between motivation and mental health in sport: A test of the hierarchical model of intrinsic and extrinsic motivation. Frontiers in Psychology, 9, 707. https://doi.org/10.3389/fpsyg.2018.00707

Smith, K. J., Davy, J. A., \& Rosenberg, D. L. (2010). An examination of the validity of the academic motivation scale with a united states business student sample. Psychological Reports, 106(2), 323-341. https://doi.org/10.2466/PR0.106.2.323-341

Sobral, D. T. (2003). Motivação do aprendiz de medicina: Uso da escala de motivação acadêmica. Psicologia: Teoria e Prática, 19(1), 25-31. Recuperado de http://www.scielo.br/pdf/\%0D/ptp/v19n1/a05v19n1.pdf. $\quad$ http://doi.org/10.1590/S010237722003000100005

Souza, Z., \& Miranda, G. (2017, outubro). (In) Estabilidade da motivação em alunos de ciências contábeis. Anais do Congresso UFU de Contabilidade, Uberlândia, MG, 2. Recuperado de: http://repositorio.ufu.br/bitstream/123456789/19919/3/\%28IN\%29EstabilidadeMotivaçãoAluno s.pdf

Stover, J. B., Bruno, F. E., Uriel, F. E., \& Liporace, M. F. (2017). Teoría de la Autodeterminación: Una revisión teórica. Perspectivas en Psicología, 14(2), 105-115. Recuperado de http://www.seadpsi.com.ar/revistas/index.php/pep/article/view/332

Thompson, B. (2000). Ten commandments of structural equation modeling. In L. G. Grimm \& P. R. Yarnold (Eds.), Reading and understanding more multivariate statistics (pp. 261-284). Washington, DC: American Psychological Association.

Vallerand, R. J., Blais, M. R., Briere, N. M., \& Pelletier, L. G. (1989). Construction et validation de I'echelle de motivation en educa- tion (EME). Canadian Journal of Behavioral, 21(3), 323-349. Retrieved http://selfdeterminationtheory.org/SDT/documents/1989_VallerandBlaisBrierePelletier_CJBSR CSC.pdf

Vanhalst, J., Luyckx, K., Petegem, S. V., \& Soenens, B. (2018). The detrimental effects of adolescents' chronic loneliness on motivation and emotion regulation in social situations. Journal of Youth and Adolescence, 47(1), 162-176. https://doi.org/10.1007/s10964-017-0686-4

Viana, G. S. (2012). Atitude e motivação em relação ao desempenho acadêmico de alunos do curso de graduação em administração em disciplinas de estatística (Dissertação de mestrado). Universidade de São Paulo, Faculdade de Economia, Administração e Contabilidade de Ribeirão Preto, Ribeirão Preto, SP, Brasil. Recuperado de http://www.teses.usp.br/teses/disponiveis/96/96132/tde-21122012-105824/pt-br.php

Viana, G. S., \& Viana, A. B. N. (2012). Attitude and motivation in relation to the academic performance of undergraduate students from statistics courses in business administration: the formation of clusters. Administração: Ensino e Pesquisa, 13(3), 523-558. Recuperado de: https://raep.emnuvens.com.br/raep/article/view/88/50 
Wolsink, I., Belschak, F. D., \& Hartog, D. N. (2018). Distraction: the problem with extrinsic motivation and proactive creativity distraction (Unpublished dissertation). University of Amsterdam, Faculty of Economics and Business, Department of leadership and management. Retrieved from https://psyarxiv.com/ctyw2/

\author{
Autores \\ Marco Ferreira Ribeiro \\ Universidade Católica Portuguesa, Viseu, 3504-505, Estrada da Circunvalação, Viseu, Portugal \\ E-mail: ferreiraribeiro.marco@gmail.com \\ Vasco Saraiva \\ Universidade Católica Portuguesa, Viseu, 3504-505, Estrada da Circunvalação, Viseu, Portugal \\ E-mail: luisvascosaraiva@gmail.com \\ Paulo Pereira \\ Universidade Católica Portuguesa, Viseu, 3504-505, Estrada da Circunvalação, Viseu, Portugal \\ E-mail: ppereira@viseu.ucp.pt \\ Célia Ribeiro \\ Universidade Católica Portuguesa, Viseu, 3504-505, Estrada da Circunvalação, Viseu, Portugal \\ E-mail: cribeiro.crb.ucp@sapo.pt
}

\title{
Contribuições
}

$1^{\circ}$ autor: Definição do problema de investigação, fundamentação teórica, definição de procedimentos metodológicos, recolha de dados, análise estatística, análise e interpretação dos dados, redação do manuscrito.

$2^{\circ}$ autor: Definição do problema de investigação, fundamentação teórica, definição de procedimentos metodológicos, recolha de dados, redação do manuscrito

$3^{\circ}$ autor: Definição do problema de investigação, definição de procedimentos metodológicos, análise estatística, análise e interpretação dos dados, redação e revisão crítica do manuscrito.

$4^{\text {o }}$ autor: Definição do problema de investigação, fundamentação teórica, definição de procedimentos metodológicos, análise e interpretação dos dados, redação e revisão crítica do manuscrito.

\section{Financiamento}

Os autores informaram que não há existência de apoio financeiro para a pesquisa neste artigo.

\section{Conflito de Interesses}

Os autores informaram que não existe conflito de interesses.

\section{Verificação de Plágio}

A RAC mantém a prática de submeter todos os documentos aprovados para publicação à verificação de plágio, mediante o emprego de ferramentas específicas, e.g.: iThenticate.

\section{Material Suplementar}

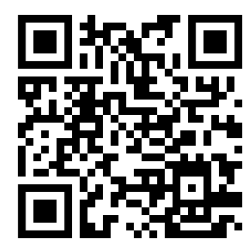

Todos os dados e materiais foram disponibilizados publicamente por meio da plataforma Mendeley e podem ser acessados em: Ribeiro, Marco Ferreira; Saraiva, Vasco; Pereira, Paulo; Ribeiro, Célia (2019), "Data for: Academic motivation scale: validity in Portuguese public higher education published by RAC-Revista de Administração Contemporânea", Mendeley Data, v1. Recuperado de http://dx.doi.org/10.17632/6n78w5pz74.1 


\section{ANEXO}

\section{Instrumento de avaliação da Motivação no Ensino Superior}

\section{Caraterização}

Sexo: $\quad$ Masculino... $\square \quad$ Feminino... $\square$ Idade:

Concelho de residência:

Estado Civil: $\quad$ Solteiro... $\square$ Casado/União de Facto... $\square \quad$ Divorciado/Separado... Outro:

É trabalhador estudante? Sim.... $\square \quad$ Não.... $\square \quad$ Tem filhos? Sim.... Quantos? Não....

Universidade: Curso:

Qual o ano que frequenta? $1^{\circ}$ Ano.... $\square \quad 2^{\circ}$ Ano.... $\square \quad 3^{\circ}$ Ano.... $\square \quad 4^{\circ}$ Ano.... $\square \quad 5^{\circ}$ Ano....

\section{Avaliação da Motivação no Ensino Superior}

\section{Porque vem à universidade?}

Com base na escala abaixo indique em que medida cada um dos itens corresponde, atualmente, a uma razão para vir à universidade.

\begin{tabular}{|c|c|c|c|c|c|c|}
\hline $\begin{array}{l}\text { Nenhuma } \\
\text { correspondência }\end{array}$ & $\begin{array}{c}\text { Pouca } \\
\text { correspondência }\end{array}$ & & pon & & $\begin{array}{c}\text { Muita } \\
\text { correspondência }\end{array}$ & $\begin{array}{c}\text { Total } \\
\text { correspondência }\end{array}$ \\
\hline 1 & 2 & 3 & 4 & 5 & 6 & 7 \\
\hline
\end{tabular}

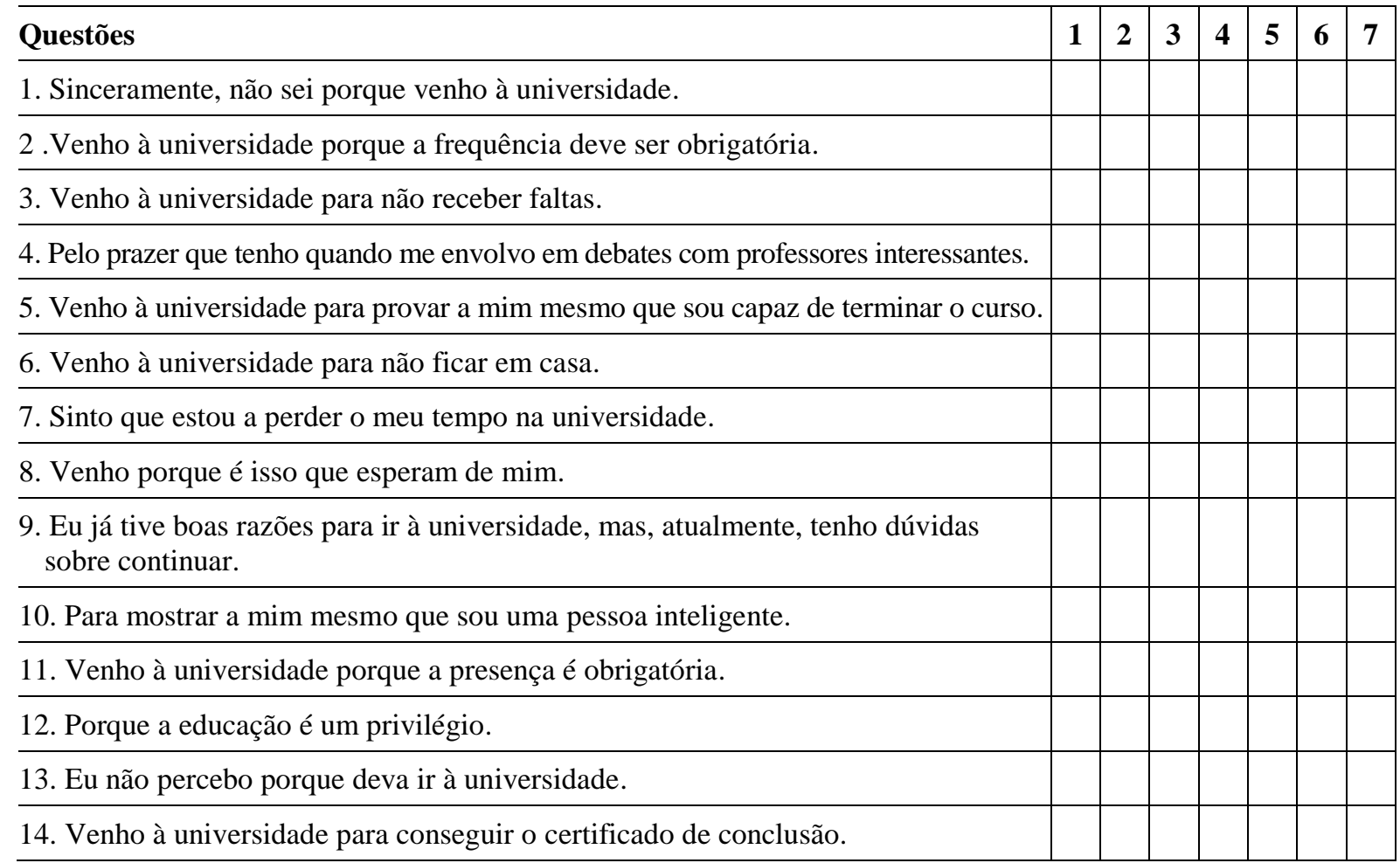


15. Venho à universidade porque quando sou bem-sucedido sinto-me importante.

16. Eu não sei, nem percebo o que estou a fazer na universidade.

17. Porque para mim a universidade é um prazer.

18. Porque o acesso ao conhecimento dá-se na universidade.

19. Eu não percebo que diferença faz vir à universidade.

20. Porque quero mostrar a mim mesmo que posso ser bem-sucedido nos estudos.

21. Porque gosto muito de vir à universidade.

22. Porque considero que o registo das presenças é necessário para a aprendizagem.

23. Quero evitar que as pessoas me vejam como um aluno desleixado.

24. Venho à universidade porque a frequência das aulas é obrigatória.

25. Caso a frequência não fosse obrigatória poucos alunos assistiriam às aulas.

26. Porque estudar amplia horizontes.

27. Venho à universidade porque foi isso que escolhi para mim.

28. Venho à universidade porque enquanto estiver a estudar não preciso de trabalhar.

29. Os meus amigos são o principal motivo pelo qual venho à universidade.

Nota. Adaptado de Guimarães, S. É. R., \& Bzuneck, J. A. (2008). Propriedades psicométricas de um instrumento para avaliação da motivação de universitários. Ciências \& Cognição, 13(1), 101-113. Recuperado de http://cienciasecognicao.org/revista/index.php/cec/article/view/682 